BI-TP 93/18

\title{
WEAK COUPLING PHASE STRUCTURE OF THE ABELIAN HIGGS MODEL AT FINITE TEMPERATURE
}

\author{
A. Jakovác ${ }^{1}$ and A. Patkós ${ }^{1,2}$ \\ ${ }^{1}$ Eötvös University, Dept. of Atomic Physics \\ H-1088 Puskin u. 5-7, Budapest, Hungary \\ ${ }^{2}$ Fakultät für Physik, Universität Bielefeld \\ Postfach 1001 31, W-4800 Bielefeld 1, Germany
}

\begin{abstract}
Using the 1-loop reduced 3D action of the Abelian Higgs-model we discuss the order of its finite temperature phase transition. A two-variable saddle point approximation is proposed for the evaluation of the effective potential. The strength of the first order case scales like $\sim e^{3-6}$. Analytic asymptotic weak coupling and numerical small coupling solutions are compared with special emphasis on the cancellation of divergences.
\end{abstract}




\section{Introduction}

The observed asymmetry between matter and anti-matter in the Universe is very probably the consequence of some out-of-equilibrium situation in the course of its History, rather than part of the initial data. [An up-to-date progress report on the subject is provided by Ref. [1].] Any starting net baryon number would have been washed out by the high-temperature B-violating processes, which are present in thermal equilibrium even in the Standard Model of electroweak interactions.

Out-of-equilibrium state of matter occurs in first order phase transitions. This circumstance has been successfully associated by Kuzmin et al. [2] with known $\mathrm{C}$ and CP violation and intense anomalous B-violation at high temperature in the Standard Model to argue that Sakharov's conditions [3] for baryogenesis can be met within a maximally known physical framework, too. Quantitative agreement with the observed asymmetry might enforce the extension of the particle content of the model enhancing the strength of CP-violating effects [4].

The present attention focused on the nature and the quantitative features of the electroweak phase transition should be appreciated on this background. The principal tool of the investigation is the effective potential at finite temperature.

In the usual loop-expansion propagators of all particles are evaluated with masses extracted from the tree-level shifted Lagrangian. For small values of the scalar field some of the mass-squares become negative, what prohibits any clean interpretation of effective potential, the interface features, etc. Summation of the class of "daisy-type", infrared sensitive contributions leads to the cancellation of the dangerous pieces [5]. A short-cut in this gradual procedure is to use the temperature corrected effective masses from the start [6]. A complete self-consistent treatment of the polarisation functions has been described by Buchmüller et al. [7] (see also [8]), where the masses are roots of a set of gap-equations.

The significance of the effective mass is obvious because the simplest signature of a second order transition comes from its vanishing. Also, the strength of a first order transition is characterised in terms of effective Lagrangians by the coefficient of the term cubic in the scalar field. This quantity is greatly influenced once again by the corrected mass of the longitudinal vector field [9].

An alternative procedure for avoiding infrared problems can be based on the idea of dimensional reduction [10]. In this approach an effective theory is derived for the static bosonic Matsubara modes, where temperature dependent masses are created by non-static modes.

In a recent publication a saddle point approximation has been proposed for the solution of the effective theory and tested on the example of the pure scalar field theory [11]. The saddle point value of some auxiliary variable serves for the effective mass-square of the scalar field. The high-temperature phase corresponds to all of its components having the same nonzero mass. Infrared problems are avoided.

A systematic expansion around the saddle point can be developed, where the 
subsequent orders are organised in powers of the inverse number of the components (large-N expansion).

Second order transitions can be approached with this method only from the symmetric phase. In case of first order transitions one can enter the broken phase down to the temperature, where the symmetric phase becomes absolutely unstable.

A closely related approach has been put forward in Ref. [12], treating the effective theory with cut-off(s). The cut-off value(s) is(are) chosen proportional to the temperature in such a way that the final expression of the effective potential should reproduce the leading high- $\mathrm{T}$ behaviour determined in the $4 \mathrm{D}$ theory. This version of the reduced field theory has been applied already to the SU(2) nonAbelian gauge theory, too [13].

In the present paper the strategy of Ref. [11] will be applied to the Abelian Higgs-model. Integration over the non-static modes will be performed at 1-loop level. In the potential piece of the effective model terms up to quartic power in the static fields are retained (higher powers being suppressed at high temperature). Both the scalar field and the "thermal" component of the vector potential are treated on equal footing. The dependence of the effective Lagrangian on the static vector field is found by exploiting the $3 \mathrm{D}$ gauge invariance of the effective theory. (Section 2)

The effective problem is solved by introducing auxiliary fields, respectively conjugate to the scalar complex field and the thermal component of the vector field. The dynamics of these fields is treated in a saddle point approximation. A simple argument shows that for any nonzero value of $e$ the transition is of first order nature in our approximation. The saddle-point equations can be solved analytically in the asymptotic weak coupling regime. (Section 3)

Finally, a numerical solution for small, but finite couplings is also presented. Here the non-trivial question of the cancellation of 3-dimensional infinities produced in the course of the derivation of effective models is carefully investigated. Also detailed comparison with other approximate treatments available in the recent litterature is given (Section 4). Conclusions are summarized in Section 5. 


\section{Derivation of the effective model}

The field theory under investigation is defined by the following Euclidean action

$$
S=-\int d^{4} x\left[\frac{1}{4} F_{m n} F_{m n}+\frac{1}{2}\left|D_{m} \Phi\right|^{2}+\frac{1}{2} m^{2}|\Phi|^{2}+\frac{\lambda}{4 !}|\Phi|^{4}\right],
$$

where $\Phi$ is a complex field and

$$
F_{m n}=\partial_{m} A_{n}-\partial_{n} A_{m}, \quad D_{m} \Phi=\left(\partial_{m}+i e A_{m}\right) \Phi, \quad m=1, . ., 4 .
$$

It is convenient to rewrite (2.1) in three-dimensional notation (the "time"components are indexed by $\tau$ ):

$$
\begin{gathered}
S=-\int d^{4} x\left[\frac{1}{4} F_{i j} F_{i j}+\frac{1}{2}\left(\partial_{\tau} A_{i}\right)^{2}+\frac{1}{2}\left(\partial_{i} A_{\tau}\right)^{2}-\left(\partial_{i} A_{i}\right)\left(\partial_{\tau} A_{\tau}\right)\right. \\
+\frac{1}{2}\left|\partial_{\tau} \Phi\right|^{2}+\frac{1}{2}\left|\partial_{i} \Phi\right|^{2}+\frac{e^{2}}{2}\left(A_{\tau}^{2}+A_{i}^{2}\right)|\Phi|^{2}+\frac{i e A_{\tau}}{2}\left(\Phi \partial_{\tau} \Phi^{*}-\Phi^{*} \partial_{\tau} \Phi\right) \\
\left.+\frac{i e A_{i}}{2}\left(\Phi \partial_{i} \Phi^{*}-\Phi^{*} \partial_{i} \Phi\right)+\frac{1}{2} m^{2}|\Phi|^{2}+\frac{\lambda}{4 !}|\Phi|^{4}\right] .
\end{gathered}
$$

The first step of the derivation is the separation of the static parts of the fields:

$$
A_{i}(\mathbf{x}, \tau)=A_{i}(\mathbf{x})+a_{i}(\mathbf{x}, \tau), \quad A_{\tau}(\mathbf{x}, \tau)=\rho(\mathbf{x})+a_{\tau}(\mathbf{x}, \tau), \quad \Phi(\mathbf{x}, \tau)=\varphi_{0}(\mathbf{x})+\varphi(\mathbf{x}, \tau)
$$

with the non-static parts fulfilling $\int a_{i} d \tau=\int a_{\tau} d \tau=\int \varphi d \tau=0$.

The purely static part of the action is a three-dimensional theory of the form

$$
\begin{gathered}
S^{(0)}=-\beta \int d^{3} x\left[\frac{1}{4} F_{i j} F_{i j}+\frac{1}{2}\left(\partial_{i} \rho\right)^{2}+\frac{1}{2}\left|\partial_{i} \varphi_{0}\right|^{2}+\frac{e^{2}}{2}\left(A_{i}^{2}+\rho^{2}\right)\left|\varphi_{0}\right|^{2}\right. \\
\left.+\frac{i e A_{i}}{2}\left(\varphi_{0} \partial_{i} \varphi_{0}^{*}-\varphi_{0}^{*} \partial_{i} \varphi_{0}\right)+\frac{1}{2} m^{2}\left|\varphi_{0}\right|^{2}+\frac{\lambda}{4 !}\left|\varphi_{0}\right|^{4}\right] .
\end{gathered}
$$

The effective theory is obtained by integrating over the non-static fields in the Gaussian approximation. For this operation it is the most convenient to work in the static thermal gauge:

$$
a_{\tau}=0
$$

Since the three-dimensional (spatial) gauge-invariance is left intact, it will be explicitly displayed by the resulting action. 


$$
\begin{aligned}
S_{a x}^{(2)}=\int d^{4} x[ & -\frac{1}{2}\left(\partial_{i} a_{j}\right)^{2}+\frac{1}{2}\left(\partial_{i} a_{i}\right)^{2}-\frac{1}{2}\left(\partial_{\tau} a_{i}\right)^{2}-\frac{1}{2}\left|\partial_{\tau} \varphi\right|^{2}-\frac{1}{2}\left|\partial_{i} \varphi\right|^{2} \\
- & \frac{1}{2} e^{2} a_{i}^{2}\left|\varphi_{0}\right|^{2}-\frac{1}{2} e^{2} \rho^{2}|\varphi|^{2}-\frac{i e}{2} \rho\left(\varphi \partial_{\tau} \varphi^{*}-\varphi^{*} \partial_{\tau} \varphi\right) \\
& -\frac{1}{2} m^{2}|\varphi|^{2}-\frac{\lambda}{4 !}\left(\varphi_{0}^{2} \varphi^{* 2}+\varphi_{0}^{* 2} \varphi^{2}+4\left|\varphi_{0}\right|^{2}|\varphi|^{2}\right) \\
& \left.-\frac{i e}{2} a_{i}\left(\varphi_{0} \partial_{i} \varphi^{*}+\varphi \partial_{i} \varphi_{0}^{*}-\varphi_{0}^{*} \partial_{i} \varphi-\varphi^{*} \partial_{i} \varphi_{0}\right)\right]
\end{aligned}
$$

In (2.7) also $A_{i}(\mathbf{x})$ is set to zero, since by the three-dimensional gauge invariance this functional dependence of the effective action can be restored from the kinetic part of the action. In this paper we neglect the "renormalisation" of the kinetic part, only the effect of the non-static modes on the potential will be discussed. (The modification of the kinetic term can be studied using the procedure described in Ref.[14]). This is the reason why we use only the $\varphi_{0}$ and $\rho$ background fields, which are set for our restricted purpose to real constants.

The most convenient form of (2.7) for the functional integration is found after several integrations by parts and by exploiting the periodicity of the small fields in $\tau$ :

$$
\begin{aligned}
S_{a x}^{(2)}=- & d d^{4} x\left\{\frac{1}{2} a_{i}\left[\left(-D_{E}+e^{2} \varphi_{0}^{2}\right) \delta_{i j}+\partial_{i} \partial_{j}\right] a_{j}\right. \\
& +\frac{1}{2} \varphi_{1}\left(-D_{E}+e^{2} \rho^{2}+m^{2}+\frac{\lambda}{2} \varphi_{0}^{2}\right) \varphi_{1} \\
& +\frac{1}{2} \varphi_{2}\left(-D_{E}+e^{2} \rho^{2}+m^{2}+\frac{\lambda}{6} \varphi_{0}^{2}\right) \varphi_{2} \\
+ & \left.e \varphi_{0} \varphi_{2} \partial_{i} a_{i}+e \rho\left(\varphi_{2} \partial_{\tau} \varphi_{1}-\varphi_{1} \partial_{\tau} \varphi_{2}\right)\right\}
\end{aligned}
$$

where the notations $D_{E}=\partial_{i}^{2}+\partial_{\tau}^{2}$ and $\varphi=\varphi_{1}+i \varphi_{2}$ are introduced. The result of the integration can be expressed in familiar Fourier-sums:

$$
\begin{gathered}
\beta V U_{e f f}\left(\rho, \varphi_{0}\right)=\beta V\left[\frac{1}{2} m^{2} \varphi_{0}^{2}+\frac{\lambda}{4 !} \varphi_{0}^{4}+\frac{e^{2}}{2} \rho^{2} \varphi_{0}^{2}\right] \\
+\sum_{n \neq 0} \sum_{k} \ln \left(k^{2}+e^{2} \varphi_{0}^{2}\right)+\frac{1}{2} \sum_{n \neq 0} \sum_{k} \ln \left(\omega_{n}^{2} k^{4}+A_{1} \omega_{n}^{2} k^{2}+A_{2} \omega_{n}^{4}+B_{1} \omega_{n}^{2}+B_{2} k^{2}+C\right),
\end{gathered}
$$

with $k^{2}=\omega_{n}^{2}+\mathbf{k}^{2}, \omega_{n}=2 \pi \beta^{-1} n$ and 


$$
\begin{gathered}
A_{1}=2 e^{2} \rho^{2}+2 m^{2}+\left(\frac{2 \lambda}{3}+e^{2}\right) \varphi_{0}^{2}, \quad A_{2}=-4 e^{2} \rho^{2}, \\
B_{1}=\left(e^{2} \rho^{2}+m^{2}+\frac{\lambda}{2} \varphi_{0}^{2}\right)\left(e^{2} \rho^{2}+m^{2}+\left(\frac{\lambda}{6}+e^{2}\right) \varphi_{0}^{2}\right)-4 e^{4} \rho^{2} \varphi_{0}^{2}, \\
B_{2}=e^{2} \varphi_{0}^{2}\left(e^{2} \rho^{2}+m^{2}+\frac{\lambda}{6} \varphi_{0}^{2}\right), \quad C=e^{2} \varphi_{0}^{2}\left(e^{2} \rho^{2}+m^{2}+\frac{\lambda}{2} \varphi_{0}^{2}\right)\left(e^{2} \rho^{2}+m^{2}+\frac{\lambda}{6} \varphi_{0}^{2}\right) .
\end{gathered}
$$

For the high- $T$ asymptotics the logarithms in (2.9) are expanded into power series, which can be stopped at $o\left(k^{-6}, \omega^{-2} k^{-4}, \ldots\right)$ since these terms would contribute $o\left(m^{2} / T^{2}\right)$ to the potential. The evaluation of the $n \neq 0$ sums is followed by that of the k-integrals $(V \rightarrow \infty)$ with a sharp cut-off $\Lambda$. Collecting the contributions up to quartic terms one finds

$$
\begin{aligned}
& U_{e f f}=\frac{1}{2} \varphi_{0}^{2}\left[m^{2}+\left(\frac{2 \lambda}{3}+3 e^{2}\right)\left(\frac{\Lambda^{2}}{8 \pi^{2}}-\frac{\Lambda}{2 \pi^{2} \beta}+\frac{1}{12 \beta^{2}}\right)+\frac{m^{2}}{8 \pi^{2}}\left(\frac{2 \lambda}{3}+e^{2}\right)-\frac{m^{2}}{4 \pi^{2}}\left(\frac{2 \lambda}{3}+3 e^{2}\right) I\right] \\
& +\frac{1}{2} \rho^{2}\left[e^{2}\left(\frac{1}{3 \beta^{2}}+\frac{m^{2}}{4 \pi^{2}}\right)-\frac{\Lambda e^{2}}{\pi^{2} \beta}\right]+\frac{e^{4} \rho^{4}}{24 \pi^{2}} \\
& +\frac{1}{2} e^{2} \rho^{2} \varphi_{0}^{2}\left[1+\left(\frac{2 \lambda}{3}+5 e^{2}\right) \frac{1}{8 \pi^{2}}-\frac{3 e^{2}}{2 \pi^{2}} I\right] \\
& +\frac{\varphi_{0}^{4}}{4 !}\left[\lambda+3\left(\frac{5}{36} \lambda^{2}+\frac{1}{2} e^{2} \lambda+\frac{3}{2} e^{4}\right)\left(\frac{1}{2 \pi^{2}}-I\right)\right] .
\end{aligned}
$$

The abbreviation $I$ denotes the logarithmically divergent integral

$$
I=\int_{0}^{\Lambda \beta} d x\left(\frac{1}{2 x}+\frac{1}{x\left(e^{x}-1\right)}-\frac{1}{x^{2}}\right) .
$$

The renormalisation prescriptions for the couplings are chosen by fixing the second derivative with respect to the scalar field to the renormalized mass, the fourth derivative to the renormalised scalar self-coupling and the coefficient of the scalar - thermal vector component vertex to the electric charge in the effective potential at $T=0$ :

$$
\begin{gathered}
m_{R}^{2}=m^{2}+\left(\frac{2 \lambda}{3}+3 e^{2}\right) \frac{\Lambda^{2}}{8 \pi^{2}}-\frac{m^{2}}{4 \pi^{2}}\left(\frac{2 \lambda}{3}+3 e^{2}\right) I+\frac{m^{2}}{8 \pi^{2}}\left(\frac{2 \lambda}{3}+e^{2}\right), \\
e_{R}^{2}=e^{2}\left[1+\left(\frac{2 \lambda}{3}+5 e^{2}\right) \frac{1}{8 \pi^{2}}-\frac{3 e^{2}}{2 \pi^{2}} I\right], \\
\lambda_{R}=\lambda+\frac{3}{\pi^{2}}\left(\frac{5}{36} \lambda^{2}+\frac{1}{6} e^{2} \lambda+\frac{3}{2} e^{4}\right)\left(\frac{1}{2}-I\right) .
\end{gathered}
$$

The renormalised effective potential takes a form, where the three-dimensional "counterterms" are displayed explicitly (below we omit the subscript $R$ from $e$ and $\lambda)$ : 


$$
\begin{aligned}
& U_{\text {eff }, R}=\frac{1}{2} \varphi_{0}^{2}\left[m_{R}^{2}+\left(\frac{2 \lambda}{3}+3 e^{2}\right) \frac{1}{12 \beta^{2}}-\left(\frac{2 \lambda}{3}+3 e^{2}\right) \frac{\Lambda}{2 \pi^{2} \beta}\right] \\
& +\frac{1}{2} e^{2} \rho^{2} \phi_{0}^{2}+\frac{1}{2} \rho^{2}\left[e^{2}\left(\frac{1}{3 \beta^{2}}+\frac{m^{2}}{4 \pi^{2}}\right)-\frac{\Lambda e^{2}}{\pi^{2} \beta}\right]+\frac{e^{4} \rho^{4}}{24 \pi^{2}}+\frac{\lambda \varphi_{0}^{4}}{4 !} .
\end{aligned}
$$

It is notable that by the gauge invariance of the full theory no quadratic divergence has appeared related to the $\rho$-field. However, in the effective model, where it plays the role of an extra scalar field, the necessity of an appropriate $\rho$-mass renormalisation is indicated by (2.14). Also the plasmon mass in front of $\rho^{2}$ is correctly reproduced.

In order to work with truly three-dimensional quantities the following rescaling is done:

$$
\begin{gathered}
U_{i}=\sqrt{\beta} A_{i}, \quad r=\sqrt{\beta} \rho, \quad \phi=\sqrt{\beta} \varphi_{0}, \\
g=\frac{\lambda}{\beta}, \quad \epsilon=\frac{e}{\sqrt{\beta}} .
\end{gathered}
$$

With this notation the final form of the 3D effective theory is written as

$$
\begin{gathered}
S_{3 D}=-\int d^{3} x\left[\frac{1}{2}\left(\left(\partial_{i} U_{j}\right)^{2}-\left(\partial_{i} U_{i}\right)^{2}\right)+\frac{1}{2}\left|\left(\partial_{i}+i \epsilon U_{i}\right) \phi\right|^{2}\right. \\
+\frac{1}{2} m_{\phi}^{2}|\phi|^{2}+\frac{1}{2}\left(\partial_{i} r\right)^{2}+\frac{1}{2} m_{r}^{2} r^{2}+\frac{g}{4 !}|\phi|^{4} \\
\left.+\frac{\epsilon^{4} \beta}{24 \pi^{2}} r^{4}+\frac{1}{2} \epsilon^{2} r^{2}|\phi|^{2}\right],
\end{gathered}
$$

where the $U_{i}\left(A_{i}\right)$-dependence is restored and the abbreviations

$$
\begin{gathered}
m_{\phi}^{2}=m_{R}^{2}+\left(\frac{2 \lambda}{3}+3 e^{2}\right) \frac{1}{12 \beta^{2}}-\left(\frac{2 \lambda}{3}+3 e^{2}\right) \frac{\Lambda}{2 \pi^{2} \beta} \\
m_{r}^{2}=e^{2}\left(\frac{1}{3 \beta^{2}}+\frac{m^{2}}{4 \pi^{2}}\right)-\frac{\Lambda e^{2}}{\pi^{2} \beta}
\end{gathered}
$$

are introduced.

Eq. (2.16) differs slightly from the effective theory of Ref.[12]. It implies the complete determination of the $r$-potential. Also the correct $T$-dependence of $m_{\phi}^{2}$ and $m_{r}^{2}$ is achieved without the somewhat artificial step of introducing different 3D momentum cut-off scales for different fields. The investigation below concentrates on the suppression of the Higgs-effect with increasing temperature. Therefore, in the next section only the spontanous creation of a vacuum expectation value for $\phi(x)$ will be investigated. However, in some physically more appealing situations, one might attempt a broader unconstrained analysis of the competing condensations of $\phi$ and $r$ in different regions of the $\left(\lambda-e^{2}\right)$-plane. 


\section{Two-variable saddle point approximation of the effective potential}

The approximate solution we are going to discuss in this section shares with other improved perturbative methods the feature of allowing some masses to take temperature dependent values. Instead of generating these expressions from summing infrared singular diagrams or from self-consistent equations for polarisation functions, we extend the model by introducing auxiliary variables, and include the contribution from their saddle-point values to the masses. The actual procedure will influence the effective masses of the scalar and the longitudinal vector components.

The auxiliary fields are introduced by the standard Hubbard -Stratonovich transformation [15] of the quartic terms in the action (2.16):

$$
\int_{C-i \infty}^{C+i \infty} d \chi \exp \left\{\frac{1}{8} \chi_{\alpha} B_{\alpha \beta} \chi_{\beta}-\frac{1}{2} \chi_{\alpha} A_{\alpha}\right\}=\text { const. } \times \exp \left\{-\frac{1}{2} A_{\alpha} B_{\alpha \beta}^{-1} A_{\beta}\right\}
$$

$(\alpha=1,2)$. In the present case the matrix $B$ and the vector $A$ are found from (2.16)

$$
A=\left(r^{2},|\phi|^{2}\right), \quad B=\frac{4}{\epsilon^{4}}\left(\frac{\beta g}{36 \pi^{2}}-1\right)^{-1}\left(\begin{array}{cc}
\frac{g}{12} & -\frac{\epsilon^{2}}{2} \\
-\frac{\epsilon^{2}}{2} & \frac{\epsilon^{4} \beta}{12 \pi^{2}}
\end{array}\right)
$$

The extended form of the 3D action reads

$$
\begin{gathered}
S_{3 D}\left[\chi_{\alpha}, \phi, U_{i}, r\right]=-\int d^{3} x\left\{\frac{1}{2}\left[\left(\partial_{i} U_{j}\right)^{2}-\left(\partial_{i} U_{i}\right)^{2}\right]+\frac{1}{2}\left|\left(\partial_{i}+i \epsilon U_{i}\right) \phi\right|^{2}\right. \\
+\frac{1}{2} M_{\phi}^{2}|\phi|^{2}+\frac{1}{2} M_{r}^{2} r^{2}+\frac{1}{2}\left(\partial_{i} r\right)^{2} \\
\left.-\frac{1}{2 \epsilon^{4}}\left(\frac{\beta g}{36 \pi^{2}}-1\right)^{-1}\left(\frac{g}{12} \chi_{1}^{2}-\epsilon^{2} \chi_{1} \chi_{2}+\frac{\epsilon^{4} \beta}{12 \pi^{2}} \chi_{2}^{2}\right)\right\}
\end{gathered}
$$

with the mass squares modified by the auxiliary fields:

$$
M_{\phi}^{2}=m_{\phi}^{2}+\chi_{2}, \quad M_{r}^{2}=m_{r}^{2}+\chi_{1} .
$$

We approximate the $\chi_{\alpha}$-integrals by real, optimised saddle-point values and evaluate the $\phi, U_{i}$ and $r$ integrals on their background. This is the standard procedure when the number of components of the scalar field is large. Here the expected nature of the $T$-dependent corrections to the $\phi$ - and $r$-masses suggests this approach. There is no formal argument for fast convergence of the expansion around this saddle. An eventual agreement with results of the improved perturbation theory could be considered as a positive sign for the adequacy of our method. 
For the 1-loop evaluation of the effective 3D potential the value of the the $\phi$-field is shifted by $\bar{\phi}$. The integration over the variables $U_{i}$ and $\phi$ are decoupled with an appropriate gauge-fixing function added to (3.3) [14]:

$$
S_{g f}=\frac{1}{2 \alpha} \int d^{3} x\left(\partial_{i} U_{i}+\alpha \epsilon \bar{\phi} \phi_{2}\right)^{2} .
$$

The corresponding Faddeev-Popov ghost contribution is

$$
U_{e f f, g h o s t}^{3 D}=\operatorname{Tr} \log \left(-\partial_{i}^{2}+\alpha \epsilon^{2} \bar{\phi}^{2}\right) .
$$

The formal result of the Gaussian functional integrations is summarised as

$$
\begin{gathered}
V U_{\text {eff }}^{(3 D)}=\frac{1}{2} \operatorname{Tr} \log \left[\delta_{i j}\left(-\partial_{k}^{2}+\epsilon^{2} \bar{\phi}^{2}\right)+\left(1-\frac{1}{\alpha}\right) \partial_{i} \partial_{j}\right]-\operatorname{Tr} \log \left(-\partial_{k}^{2}+\alpha \epsilon^{2} \bar{\phi}^{2}\right) \\
+\frac{1}{2} \operatorname{Tr} \log \left(-\partial_{k}^{2}+M_{r}^{2}\right)+\frac{1}{2} \operatorname{Tr} \log \left(-\partial_{k}^{2}+M_{\phi}^{2}\right)+\frac{1}{2} \operatorname{Tr} \log \left(-\partial_{k}^{2}+M_{\phi}^{2}+\alpha \epsilon^{2} \bar{\phi}^{2}\right) \\
+V\left[\frac{1}{2} M_{\phi}^{2} \bar{\phi}^{2}+\frac{1}{2 \epsilon^{4}}\left(1-\frac{\beta g}{36 \pi^{2}}\right)^{-1}\left(\frac{g}{12} \chi_{1}^{2}+\frac{\epsilon^{4} \beta}{12 \pi^{2}} \chi_{2}^{2}-\epsilon^{2} \chi_{1} \chi_{2}\right)\right] .
\end{gathered}
$$

The traces in the above expression were evaluated with sharp cut-off $\Lambda_{3}$ :

$$
\begin{gathered}
U_{\text {eff }}^{(3 D)}=\Lambda_{3} \frac{1}{4 \pi^{2}}\left(2 \epsilon^{2} \bar{\phi}^{2}+M_{r}^{2}+2 M_{\phi}^{2}\right)+\frac{1}{2} M_{\phi}^{2} \bar{\phi}^{2} \\
+\frac{1}{2 \epsilon^{4}}\left(1-\frac{\beta g}{36 \pi^{2}}\right)^{-1}\left(\frac{g}{12} \chi_{1}^{2}+\frac{\epsilon^{4} \beta}{12 \pi^{2}} \chi_{2}^{2}-\epsilon^{2} \chi_{1} \chi_{2}\right) \\
-\frac{1}{12 \pi}\left[-\left(\alpha \epsilon^{2} \bar{\phi}^{2}\right)^{3 / 2}+2 \epsilon^{3}|\bar{\phi}|^{3}+M_{r}^{3}+M_{\phi}^{3}+\left(M_{\phi}^{2}+\alpha \epsilon^{2} \bar{\phi}^{2}\right)^{3 / 2}\right] .
\end{gathered}
$$

The finiteness of the expression in the third line of (3.8) requires the renormalisation of the $\chi_{\alpha}$ values [11] as seen from the detailed expressions of $M_{\phi}^{2}$ and $M_{r}^{2}$ :

$$
\begin{gathered}
M_{\phi}^{2}=m_{\phi, R}^{2}-\left(\frac{2 \lambda}{3}+3 e^{2}\right) \frac{\Lambda}{2 \pi^{2} \beta}+\chi_{2}, \\
M_{r}^{2}=m_{r, R}^{2}-\frac{\Lambda e^{2}}{\pi^{2} \beta}+\chi_{1}, \\
m_{\phi, R}^{2}=m_{R}^{2}+\left(\frac{2 \lambda}{3}+3 e^{2}\right) \frac{1}{12 \beta^{2}}, \quad m_{r, R}^{2}=e^{2}\left(\frac{1}{3 \beta^{2}}+\frac{m_{R}^{2}}{4 \pi^{2}}\right) .
\end{gathered}
$$

The cancellation of the infinities implies the existence of the cut-off independent saddle-points

$$
\chi_{1 R}=\chi_{1}-\frac{\Lambda e^{2}}{\pi^{2} \beta}, \quad \chi_{2 R}=\chi_{2}-\left(\frac{2 \lambda}{3}+3 e^{2}\right) \frac{\Lambda}{2 \pi^{2} \beta} .
$$


One reexpresses the $\chi$-dependent part of (3.8) and throws away the (infinite) constants arising in this way. Below, we write down in separate expressions the linearly divergent and the finite parts of the effective potential after reintroducing $4 \mathrm{D}$ notations. For the simplification of the expressions from this point we use the $\alpha=0$ gauge.

$$
\begin{gathered}
U_{\text {eff }, \text { div }}=\Lambda_{3} \frac{1}{4 \pi^{2}}\left(2 e^{2} \bar{\Phi}^{2}+\chi_{1 R}+2 \chi_{2 R}\right) \\
-\Lambda \frac{1}{2 e^{2}}\left(1-\frac{\lambda}{36 \pi^{2}}\right)^{-1}\left[\chi_{1 R} \frac{1}{2 \pi^{2}}\left(\frac{\lambda}{3}+3 e^{2}\right)-\chi_{2 R} \frac{e^{2}}{\pi^{2}}\left(\frac{1}{12 \pi^{2}}\left(\frac{2 \lambda}{3}+3 e^{2}\right)-1\right)\right] \\
U_{\text {eff,finite }}=\frac{1}{2}\left[m_{R}^{2}+\left(\frac{2 \lambda}{3}+3 e^{2}\right) \frac{T^{2}}{12}+\chi_{2 R}\right] \bar{\Phi}^{2} \\
+\frac{1}{2 e^{4}}\left(1-\frac{\lambda}{36 \pi^{2}}\right)^{-1}\left(\frac{\lambda}{12} \chi_{1 R}^{2}+\frac{e^{4}}{12 \pi^{2}} \chi_{2 R}^{2}-e^{2} \chi_{1 R} \chi_{2 R}\right) \\
-\frac{T}{12 \pi}\left[2 e^{3} \bar{\Phi}^{3}+\left(e^{2}\left(\frac{T^{2}}{3}+\frac{m_{R}^{2}}{4 \pi^{2}}\right)+\chi_{1 R}\right)^{3 / 2}+2\left(m_{R}^{2}+\left(\frac{2 \lambda}{3}+3 e^{2}\right) \frac{T^{2}}{12}+\chi_{2 R}\right)^{3 / 2}\right] .
\end{gathered}
$$

The vanishing of (3.11) should be automatic, when $\chi_{\alpha, R}(\bar{\Phi})$ extremising $(3.12)$ is substituted into it. The only freedom we have is to choose $\Lambda_{3}$ in constant proportion to $\Lambda$. A sufficient condition for this is to find

$$
\chi_{\alpha R}=c_{\alpha 1}+c_{\alpha 2} \bar{\Phi}^{2}
$$

since the field-independent parts of $U_{\text {eff,div }}$ can be omitted. The fulfillment of (3.13) should be checked for any solution to be presented below.

The saddle point coordinates should be determined from the following extremal conditions:

$$
\begin{gathered}
\frac{\partial U_{\text {eff,finite }}}{\partial \chi_{1 R}}=-\frac{T}{8 \pi}\left[m_{r, R}^{2}+\chi_{1 R}\right]^{1 / 2}+\frac{1}{2 e^{4}}\left(1-\frac{\lambda}{36 \pi^{2}}\right)^{-1}\left(\frac{\lambda}{6} \chi_{1 R}-e^{2} \chi_{2 R}\right)=0, \\
\frac{\partial U_{\text {eff }, \text { finite }}}{\partial \chi_{2 R}}=\frac{1}{2} \bar{\Phi}^{2}+\frac{1}{2 e^{2}}\left(1-\frac{\lambda}{36 \pi^{2}}\right)^{-1}\left(\frac{e^{2}}{6 \pi^{2}} \chi_{2 R}-\chi_{1 R}\right)-\frac{T}{4 \pi}\left[m_{\phi, R}^{2}+\chi_{2 R}\right]^{1 / 2}=0 .
\end{gathered}
$$

The location of the non-trivial minima of the effective potential is determined by the equation

$$
\frac{\partial U_{\text {eff }, \text { finite }}}{\partial \bar{\Phi}^{2}}=\frac{1}{2}\left[m_{\phi, R}^{2}+\chi_{2 R}(\bar{\Phi})\right]-\frac{e^{3} T}{4 \pi} \bar{\Phi}=0 .
$$


The phase structure in the $\lambda-e^{2}$ plane

It is not difficult to argue that eqs.(3.14-15) imply a first order transition for any non-zero value of $e^{2}$.

From the first equality in eq.(3.14) for $e^{2} \rightarrow 0$ one realizes that $\chi_{1 R}$ and $\chi_{2 R}$ are related to each other for all values of $\bar{\Phi}$ :

$$
\frac{\lambda}{6} \chi_{1 R} \approx e^{2} \chi_{2 R} .
$$

Using this relation in the second equality one solves it for the full "effective" mass

$$
H^{1 / 2}=\left[\chi_{2 R}+m_{\phi, R}^{2}\right]^{1 / 2}
$$

leading to

$$
H^{1 / 2}=\frac{1}{2}\left[-\frac{\lambda T}{12 \pi}+\left(\frac{\lambda^{2} T^{2}}{(12 \pi)^{2}}+4\left(\frac{\lambda}{6} \bar{\Phi}^{2}+m_{\phi, R}^{2}\right)\right)^{1 / 2}\right] .
$$

Defining the temperature $T_{2}$, where the symmetric minimum becomes absolutely unstable $(H(\bar{\Phi}=0)=0)$, a simple expression is derived for $H\left(\bar{\Phi}, T_{2}\right)$ :

$$
H(\bar{\Phi})^{1 / 2}=\frac{1}{2}\left[-\frac{\lambda T_{2}}{12 \pi}+\left(\frac{\lambda^{2} T_{2}^{2}}{(12 \pi)^{2}}+\frac{2 \lambda}{3} \bar{\Phi}^{2}\right)^{1 / 2}\right] .
$$

After substituting (3.19) into (3.15) one sees the origin transformed into local maximum, while a non-trivial minimum is found at

$$
\bar{\Phi}_{\text {min }}=\frac{e T_{2}}{2 \pi}+o\left(e^{2} T_{2}\right) .
$$

This behaviour has been confirmed by the numerical solution of (3.14-15) for $\lambda=10^{-5}$. It is interesting to note that Fig. 1 also illustrates the difficulty of such test. The solution of (3.14) with restricted accuracy leads to an apparent stopping of the position of $\Phi_{\min }$ when the charge $e$ becomes small enough. With gradually improved accuracy one observes the formation of a linear envelope in the interval $e \in\left(10^{-7}, 10^{-3}\right)$.

The form of $U_{\text {eff }}\left(T_{2}\right)$ shows that for $T_{c}>T_{2}$ the distance between the origin and the non-trivial minimum is finite, the transition is of first order nature. However, the effect of contributions from fluctuations around the saddle point might change this conclusion.

\section{The asymptotic weak coupling solution}

A fully analytic selfconsistent construction will be presented for the solution of the gap equations when $\lambda<<1, e^{2}<<1$. Special attention will be paid to the cancellation of the linear divergencies. 
It is convenient to introduce the notations

$$
m_{e f f}^{2}=m_{\phi, R}^{2}-\frac{e^{2} T m_{r, R}}{4 \pi}
$$

and

$$
Q=\frac{\lambda}{6}-\frac{e^{4} T}{8 \pi m_{r, R}}\left(1-\frac{\lambda}{36 \pi^{2}}\right)
$$

The temperature range covered by our analysis will be controlled by requiring

$$
m_{e f f}^{2} \sim o\left(e^{n} T^{2}\right)
$$

From the previous subsection it is clear, that $T_{c}^{2}>T_{2}^{2} \approx-36 m_{R}^{2} /\left(2 \lambda+9 e^{2}\right)$. Therefore, $m_{r, R} \sim o(e T)$.

Our fundamental assumption is that in the relevant $T$-interval $\chi_{1}<<m_{r, R}^{2}$, which will be checked on the solution. Under this assumption we expand the squareroot of the first equation of (3.14), and find an approximate linear relationship between $\chi_{1}$ and $\chi_{2}$. Using it in the second equation of (3.14) one writes for $m_{\phi, R}^{2}+$ $\chi_{2 R}$ :

$$
m_{\phi, R}^{2}+\chi_{2 R}+\frac{Q T}{2 \pi} \sqrt{m_{\phi, R}^{2}+\chi_{2 R}}-\left(Q \bar{\Phi}^{2}+m_{e f f}^{2}\right)+o\left(\frac{e^{2} T \chi_{1 R}}{m_{r, R}^{3}}, \frac{e^{4} T \chi_{2 R}}{m_{r, R}}\right)=0 .
$$

Its solution yields

$$
\begin{gathered}
\chi_{1 R}=-\frac{\lambda e^{4} T m_{r, R}}{144 \pi^{3} Q}+\frac{Q e^{2} T^{2}}{8 \pi^{2}}+e^{2} \bar{\Phi}^{2} \\
-\frac{e^{2} T}{2 \pi}\left[\frac{Q^{2} T 2}{16 \pi^{2}}+Q \bar{\phi}^{2}+m_{e f f}^{2}\right]^{1 / 2}+o\left(\frac{e^{4} T \chi_{1 R}}{Q m_{r, R}^{3}}, \frac{e^{6} T \chi_{2 R}}{Q m_{r, R}}\right), \\
\chi_{2 R}=-\frac{e^{2} T m_{r, R}}{4 \pi}+\frac{Q^{2} T^{2}}{8 \pi^{2}}+Q \bar{\Phi}^{2} \\
-\frac{Q T}{2 \pi}\left[\frac{Q^{2} T^{2}}{16 \pi^{2}}+Q \bar{\Phi}^{2}+m_{e f f}^{2}\right]^{1 / 2}+o\left(\frac{e^{2} T \chi_{1 R}^{2}}{m_{r, R}^{2}}, \frac{e^{4} T \chi_{2 R}}{m_{r, R}}\right) .
\end{gathered}
$$

The range of $\bar{\Phi}$, where this solution is required to be valid should cover the anticipated second non-trivial minimum of the potential. The order of magnitude of its distance from the origin can be estimated with help of (3.15), when the above expression of $\chi_{2}$ is substituted:

$$
m_{e f f}^{2}+Q \bar{\Phi}_{m i n}^{2}-\left(\frac{e T}{2 \pi}\right)^{3 / 2} \frac{Q}{2} \bar{\Phi}_{m i n}^{1 / 2}-\frac{e^{3} T}{2 \pi} \bar{\Phi}_{m i n}=0
$$


That part of the $\lambda-e^{2}$-plane will be considered, where terms proportional to $Q$ can be neglected in the equation of $\bar{\Phi}_{\min }$ :

$$
\bar{\Phi}_{\text {min }}=\frac{2 \pi m_{e f f}^{2}}{e^{3} T} \sim o\left(e^{n-3} T\right) .
$$

Since the requirement $\chi_{1 R}<<m_{r, R}^{2}$ implies $\bar{\Phi}<<T$, one has to require $n>3$. A posteriori checking the order of magnitude of the omitted terms in (3.25), the consistency gives the condition

$$
Q<o\left(e^{m}\right), \quad m=\max \left(6-n, \frac{n}{2}\right) .
$$

This estimate can be used for further simplifying the expressions of the saddle point values:

$$
\begin{gathered}
\chi_{1 R}=-\frac{e^{2} T}{2 \pi} m_{e f f}+e^{2} \bar{\Phi}^{2}+o\left(Q^{2} T^{2}\right), \\
\chi_{2 R}=-\frac{e^{2} T}{4 \pi} m_{r, R}-\frac{Q T}{2 \pi} m_{e f f}+Q \bar{\Phi}^{2}+o\left(Q^{2} T^{2}\right) .
\end{gathered}
$$

The order of magnitude estimate of the errors in (3.28) allows for $n$ the range $3<n<4$. It is this range which fixes, how close we can tune our solution to the $m_{\text {eff }}=0$ temperature. (It is worth to notice that from here by the definition of $Q$ also $\lambda \sim o\left(e^{3}\right)$ follows. Also one explicitly sees that $\chi_{1 R}$ is negligible relative to $m_{r, R}^{2}$.)

Before going to the description of the behaviour of $U_{\text {eff }}$, we consider (3.11), the linearly divergent piece of the potential. One finds that choosing

$$
\Lambda_{3} \approx \Lambda\left(1+\frac{\lambda}{9 e^{2}}\right)
$$

its cancellation is ensured, since our solution (3.28) is conform with (3.13).

The leading expression of the potential then is given by

$$
U_{e f f}=\frac{1}{2} m_{e f f}^{2} \bar{\Phi}^{2}-\frac{T}{6 \pi} e^{3} \bar{\Phi}^{3}+\frac{Q}{4} \bar{\Phi}^{4}
$$

Up to the modification $\lambda / 6 \rightarrow Q$ this expression coincides with the improved form derived by Arnold [6]. It has been checked on the numerical solution $\left(\lambda=10^{-5}, e^{2}=10^{-4}\right)$ that the solution is very well represented by the above formulae. Note, however, that the only reasonable comparison was to calculate the effective potentials at the transition temperatures of the respective approximations.

The weakening of the strength of the first order transition for small $e$ can be characterised by the scaling of the height of the barrier between the degenerate minima at $T_{c}$. From (3.30) one finds the power $e^{3 n-6}$. In view of the allowed range of $n$ this leads to a "height-exponent" between 3 and 6 . 


\section{Small coupling numerical solution}

The solution of the system (3.14) exists whenever the squre roots appearing in these equations take real values. We have explicitly found $\chi_{\alpha, R}$ in the full $\bar{\Phi}$ interval covering both minima of the potential for the coupling space $10^{-7} \leq \lambda \leq$ $10^{-1}, 10^{-6} \leq e^{2} \leq 0.5$. In the whole region the symmetry restoration proceeds through first order transition. The situation is well illustrated by Fig.2, where also curves of the effective potential below and above $T_{c}$ are displayed. All quantities are displayed in proportion to an appropriate power of the $T=0$, tree level expectation value of $\bar{\Phi}$. The complex nature of the effective potential expected in intervals of non-convexity does not show up in the present approximation.

Our solution for these not asymptotically small couplings $\left(\lambda=0.07, e^{2}=.32\right)$ clearly deviates from the weak coupling solution of the previous section. The measure of the deviation can be quantitatively assessed by Fig.3, where the numerically obtained $\chi_{2 R}$ is displayed together with (3.28). The deviation is small near $\bar{\Phi}=0$, but increases gradually towards larger $\bar{\Phi}$. It is notable that $\chi_{2}$ yields a negative contribution to the effective mass of $\bar{\Phi}$ near the origin. This represents a tendency towards the instability of a homogenous background expected for small values of the field and for temperatures below the appearance of the non-trivial metastable local minimum. In case of $\chi_{1 R}$ the deviation of the numerical solution from the asymptotic weak coupling formulae cannot be resolved visually on the same scale.

From the point of view of selfconsistency the cancellation of the linearly divergent piece should be investigated. Any deviation from zero would point to the importance of corrections to the saddle point approximation. In Fig.4 the coefficient of $\Lambda_{3}$ in eq.(3.11) is displayed $(\Gamma)$ against the same quantity in front of $\Lambda(\Delta)$. The observed fully linear behaviour makes our leading approximation selfconsistent in an extended part of the $\left(\lambda, e^{2}\right)$ plane. The slope is found extremely close to unity, supporting the validity of the weak coupling relation (3.20).

Our results can be compared quantitatively with curves of the effective potential published in [7] for not asymptotically small couplings. The values $\lambda=$ $0.15, e^{2}=0.25$ correspond to Fig. 8 of Ref [7]. The temperature closest to the actual transition, which can be faithfully described perturbatively according to the authors is $T^{*}=T_{V}$ (in their notation), the temperature where the infrared sensitivity of the vector contribution to the potential becomes important. Its value for the above coupling point is $T_{V}=0.647$. Since there the deviation of $U_{\text {eff }}\left(0, T^{*}\right)$ from $U_{\text {eff }}\left(\Phi_{\text {min }}, T^{*}\right)$ is at the $10^{-10}$ level, $T^{*}$ for all practical purposes can be considered as $T_{c}$ of the approximation scheme [7] in this point. Our method leads for the same couplings to $T_{c}=0.646$. The barrier height between degenerate minima at $T_{c}$ in proportion of the fourth power of the $T=0$, classical vacuum expectation value of the Higgs field in the respective approximations is $U_{\text {eff }}\left(\bar{\Phi}_{\max }, T_{c}\right)=1.76 \times 10^{-5}[7], 1.48 \times 10^{-5}$ (present work). Finally, the Higgs expectation value at the transition in proportion to its $T=0$, classical value is $\bar{\Phi}_{\min }\left(T_{c}\right)=0.492[7], 0.463$ (present work). 


\section{Conclusions}

In this paper we have described in detail a two-variable saddle-point improvement of the 1-loop finite temperature effective potential of the Abelian Higgs-model. Its main advantage is that it avoids the need to discuss explicitly the delicate problem of infrared sensitivity. On the other hand it is quite complicated to go beyond the leading saddle-point approximation in the Abelian case compared to the pure scalar model with $N=\infty$ components.

Nevertheless the practical renormalisability of the effective potential beyond asymptotically small couplings allows a rather clean interpretation of the leading order results beyond the domain of its strict applicability. The good quantitative agreement with more standard perturbative improvements gives confidence in applying the method to more relevant systems, too. Explicit calculations of the $o\left(e^{2}, \lambda^{2}\right)$ corrections in the scheme of [7], and the evaluation of the fluctuations around the saddle point in the present approximation might decide if this spectacular agreement is more than just an accident.

The first order transition we observe is extremely weak. The barrier between the degenerate minima at the transition seems to scale like $\sim e^{3-6}$.

\section{Acknowledgements}

The authors thank W. Buchmüller for kindly providing some data for comparing the present method with his selfconsistent improved perturbative approach. Also remarks by F. Karsch and J. Polonyi are gratefully acknowledged. The research of A.P. was partly supported by the EC-grant ERB3510PL920739.

\section{Figure Captions}

Fig.1 Shifting of the position of the non-trivial minimum of the effective potential at $T_{2}$ with decreasing $e$ as found from the numerical solution. The leveling off is the result of the finite accuracy of the solution. The true behavior as it is predicted by the weak coupling analysis is given by the common envelope.

Fig.2 The effective potential for $\lambda=0.07$ and $e^{2}=0.32$. The three curves illustrate the ability of our method to describe the neighbourhood of the transition in both phases

Fig.3 Comparison of the weak coupling and the numerical solutions of $\chi_{2 R}$ in the same $\lambda, e^{2}$ point as for Fig.2 $\left(T=T_{c}\right)$. The curve 1 gives the numerical solution for $\chi_{2}$, curve 2 represents its weak coupling solution (3.28).

Fig.4 Illustration of the linear relation between $\Lambda_{3}$ and $\Lambda$ for couplings as above. The coefficient of $\Lambda_{3}$ is denoted by $\Gamma$, that of $\Lambda$ by $\Delta\left(T=T_{c}\right)$, 


\section{References}

1. A.G. Cohen, D.B. Kaplan and A.E.Nelson Progress in Electroweak Baryogenesis, UCSD-PTH-93-02, BUHEP-93-4

2. V.A. Kuzmin, V.A. Rubakov and M.E. Shaposhnikov, Phys. Lett. 155B (1985) 36

3. A.D. Sakharov, JETP Letters 5 (1967) 24

4. L. McLerran, Phys. Rev. Lett. 62 (1989) 1075

5. L. Dolan and R. Jackiw, Phys. Rev. D9 (1974) 3320

S. Weinberg, Phys. Rev. D9 (1974) 3357

6. M.E. Carrington, Phys. Rev. D45 (1992) 2933

P. Arnold, Phys. Rev. D46 (1992) 2628

7. W. Buchmüller, T. Helbig and D. Walliser, First Order Transitions in Scalar Electrodynamics, DESY-92-151, and DESY-93-021

8. J.R. Espinosa, M. Quirós and F. Zwirner, Phys. Lett. 191B (1992) 115 and CERN-TH.6577/92

9. M. Dine, R.L. Leigh, P. Huet, A. Linde and D. Linde, Phys. Rev. D46 (1992) 550

10. T. Appelquist and R. Pisarski, Phys. Rev D23 (1981) 2305

N.P. Landsman, Nucl. Phys. B322 (1989) 498

11. H. Meyer-Ortmanns and A. Patkós, Phys. Lett. 297B (1993) 321

12. V. Jain The 3D Effective Field Theory of the High Temperature Abelian Higgs Model, MPI-Ph/92-72

13. V. Jain and A. Papadopolous, Phys. Lett. 303B (1993) 315

14. I. Moss, D. Toms and A. Wright, Phys. Rev. D46 (1992) 1671

15. R.L. Stratonovich, Dokl. Akad. Nauk SSSR 115 (1957)

J. Hubbard, Phys. Rev. Lett. 3 (1959) 77 